"NOTICE: this is the author's version of a work that was accepted for publication in Measurement Science and Technology. Changes resulting from the publishing process, such as peer review, editing, corrections, structural formatting, and other quality control mechanisms may not be reflected in this document. Changes may have been made to this work since it was submitted for publication. A definitive version will be subsequently published in Measurement Science and Technology. [VOL17, pp.797-808, (2006)].”

\title{
Confocal micro-PIV measurements of three dimensional profiles of cell suspension flow in a square microchannel
}

\author{
R. LIMA ${ }^{1,2}$, S. WADA ${ }^{1}$, K. TSUBOTA ${ }^{1}$, T. YAMAGUCHI ${ }^{1}$ \\ ${ }^{1}$ Dept. Bioeng. \& Robotics, Grad. Sch. Eng., Tohoku Univ., 6-6-01 Aoba, 980-8579 Sendai, Japan. \\ ${ }^{2}$ Dept. Mechanical Eng., ESTiG, Bragança Polyt., C. Sta. Apolónia, 5301-857 Bragança, Portugal. \\ E-mail: rui@pfsl.mech.tohoku.ac.jp
}

\begin{abstract}
The detail measurements of velocity profiles of in vitro blood flow in microchannels is fundamental for a better understanding on the biomechanics of the microcirculation. It is therefore very important to obtain measurements with high accuracy and spatial resolution of the influence of the blood suspension cells on the flow behaviour. This paper presents and compares measurements of pure water and suspension of blood cells diluted in a physiological fluid within a square microchannel obtained by a confocal particle image velocimetry (PIV) system. This emerging technology by combining the conventional PIV system with a spinning confocal microscope has the ability to obtain not only high spatial resolution images but also three dimensional (3D) optical sectioning velocity measurements. The good agreement obtained between measured and estimated results suggests that macroscale flow theory can be used to predict the flow behaviour of a homogenous fluid within a $100 \mu \mathrm{m}$ square microchannel. Our results also have demonstrated the potentiality of our system to generate $3 D$ profiles and consequently to obtain detail information about micro-scale effects in microchannels by using both homogeneous and non-homogeneous fluids such as suspension of blood cells in the flow. Furthermore, our confocal micro-PIV system by employing the new scanning unit CSU-22, developed by Yokogawa, made it possible for the first time to measure successfully velocities up to $0.52 \mathrm{~mm} / \mathrm{s}$ of a blood cell suspension fluid.
\end{abstract}

Key words: microcirculation, confocal micro-PIV, Nipkow disk, blood cell suspension, microchannel.

\section{Introduction}

The phenomena of blood flow in microcirculation (microvessels with diameter less than $250 \mu \mathrm{m}$ ) is characterized mainly by the red blood cells (RBCs) flow behaviour, which might be normal or pathological. Despite the high amount of research in microcirculation, there has not yet been any detailed experimental information about flow velocity profiles, RBCs deformability and aggregation in microvascular networks (Lee 2000, Mchedlishvili and Maeda 2001, Lipowsky 2005). This lack of knowledge was mainly due to the absence of adequate techniques with high spatial and temporal resolution to measure and quantitatively evaluate fluid mechanical effects at a microscopic level.

During the years the most research work in this area has focused in experimental studies using optical techniques mainly because they are less invasive to measure the flow field when compared with other methods. One of the most popular optical technique is the particle-based flow velocimetry, where trace particles are seeded to the flow. In order to acquire and analyse the particle data there are several techniques such as laser Doppler velocimetry (LDV), particle streak velocity (PSV) and particle image velocimetry (PIV) (Sinton 2004). The most practical and commonly used method is the PIV method which is a well establish technique to measure macroscopic fluidics (Raffel et al. 1998). Recently, this technique has been successfully extended to micro-scale flows by combining the conventional PIV system with an inverted epi-fluorescent microscope (Santiago et al. 1998). This combination, known as micro-PIV, have greatly increased the resolution of the conventional PIV and as a result the micro-PIV technique have started to be widely used to investigate the flow behaviour in micro-fluidic devices. (Meinhart et al. 1999, Koutsiaris et al. 1999, Sugi et al. 2002, klank et al. 2002, Devasenathipathy et al. 2003, Chiu et al.2003, Shinohara et al. 2004). However, by using conventional microscopes the entire 
flow field is illuminated and consequently the out-of-focus emitted light can result in high levels of background noise which contribute to degrading the measured velocity fields (Meinhart et al. 1999, Meinhart et al. 2000, Nguyen and Wereley 2002).

More recently, considerable progress in the development of confocal microscopy and consequent advantages of this microscope over the conventional microscopes (Pawley 1990, Wright and Wright 2002, Inoue and Inoue 2002, Amos and White 2003) have led to a new technique known as confocal micro-PIV (Tanaami et al. 2002, Kinoshita et al. 2005, Lima et al. 2005) or as confocal laser scanning microscopy (CLSM) micro-PIV (Park et al. 2004). This technique combines the conventional PIV system with a spinning disk confocal microscope (SDCM). Due to its outstanding spatial filtering technique together with the multiple point light illumination system, this kind of microscope have the ability to obtain infocus images with optical thickness less than $1 \mu \mathrm{m}$ (optical sectioning effect), which is a task extremely difficult to be achieved by using a conventional microscope. As a result, by combining SDCM with the conventional PIV system it is possible to achieve a PIV system with not only extremely high spatial resolution but also with capability to generate 3D velocity profiles. Very recently, Park and his colleagues (Park etal. 2004) have compared his confocal micro-PIV with a conventional micro-PIV. This study has demonstrated that confocal micro-PIV system improves the particle image contrasts, definitions and accuracy of the velocity measurements. However, in their work they did not explore the optical sectioning capability to generate 3D velocity profiles and more importantly they have always carried out their experiments by using a homogenous fluid. According to our knowledge there has not yet been any study in the literature that evaluates the ability of a confocal micro-PIV system to investigate phenomena in a non-homogenous fluids such as fluids containing suspension of cells. It should also be noted that the system used by Park et al. had very high spatial accuracy but its temporal resolution was very low (up to 120 frames per second) which limits the applicability of their system to study phenomena in microcirculation. In contrast, our system uses the new scanning unit CSU22, developed by Yokogawa, which enables to acquire confocal images up to 2000 frames per second. This new upgraded confocal micro-PIV system enables researchers to quantify the flow patterns inside microchannels with high spatial and temporal resolution.

The aim of the present study is to evaluate the performance of our confocal micro-PIV system in order to investigate its ability to study the behaviour of a non-homogenous fluids within a square microchannel. In addition, the current work provides the first attempt to obtain 3D profiles from the optical sectioning capability of confocal micro-PIV systems.

\section{Materials and methods}

\subsection{Working fluids}

It is general practice to use a well known Newtonian fluid to evaluate the performance of a new measurement technique. In this study pure water (PW) was used as our working reference fluid in order to analyse the performance of the confocal micro-PIV system. The flow measurements through the microchannel was conducted by seeding particles which follow the flow. These particles should scatter light efficiently and should be small enough when compared with the dimensions of the microchannel. However, to avoid Brownian motion the particles should be bigger than $500 \mathrm{~nm}$ (Santiago et al. 1998; Nguyen and Wereley 2002, Devasenathipathy et al. 2003). In this way, by selecting particles with a nominal diameter of $1 \mu \mathrm{m}$ we expect to reduce significantly the effect of Brownian motion.

Two working fluids were used in this study. The first was PW seeded with $0.1 \%$ (by volume) of $1 \mu \mathrm{m}$ diameter red fluorescent solid polymer microspheres (R0100, Duke Scientific). A second fluid was Hanks solution (HS) seeded with $10 \%$ (by volume) of human blood and $0.1 \%$ (by volume) of $1 \mu \mathrm{m}$ diameter red fluorescent solid polymer microspheres (R0100, Duke Scientific). It should be mentioned that the blood was collected from the venous of a healthy addult and heparine was added into it in order to prevent coagulation of the blood cells. Moreover, the blood was hermetical stored at about $4^{\circ} \mathrm{C}$ until the performance of the experiment at room temperature $\left(27^{\circ} \mathrm{C}\right)$. 


\subsection{Square microchannel}

Rectangular microchannels have become a popular in vitro technique to study blood flow phenomena under physiological flow conditions. In addition, it is well known that laminar flow through this kind of channels generates a purely axial flow. As a result, it is common practice to evaluate the performance of a new measurement flow technique by using rectangular microchannels (Brown 2000, Meinhart et al. 1999, Kroll et al. 1996). However, in our study we have decided to use a $100 \mu \mathrm{m} \times 100 \mu \mathrm{m}$ borosilicate glass square microchannel fabricated by Vitrocom as the flow behaviour is much closer to flow trough capillaries. A schematic of the tested square microchannel is shown is figure 2 . The microchannel was mounted on a slide glass with thickness of approximately $120 \mu \mathrm{m}$ which was immersed in pure water in order to minimize some possible refraction from the walls of the microchannel.

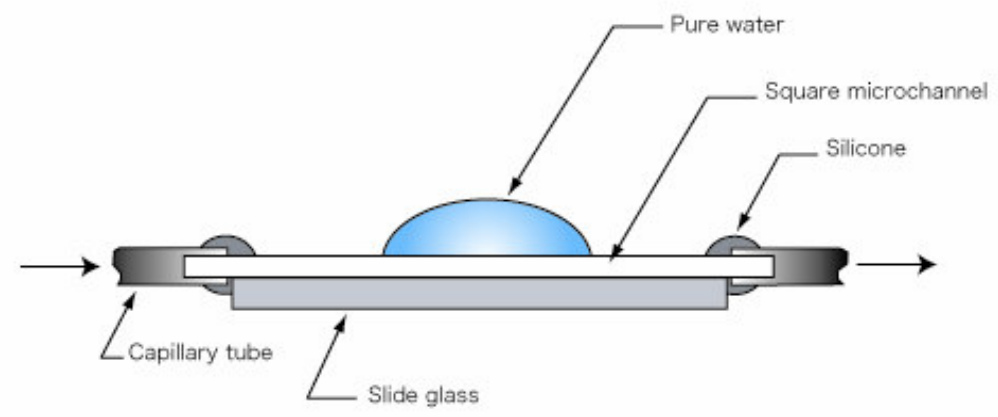

Figure 1. Diagram showing the components of the square microchannel device.

\subsection{Confocal micro-PIV system and experimental setup}

Our system is a combination of a confocal spinning disk microscope with a conventional PIV technique. For simplicity we have adopted our system as confocal micro-PIV. This system is also known by some researchers as confocal laser scanning microscopy (CLSM) micro-PIV (Park et al. 2003).The confocal micro-PIV system used in our experiment consists of an inverted microscope (IX71, Olympus, Japan) combined with a confocal scanning unit (CSU22, Yokogawa, Japan) and a diode-pumped solid state (DPSS) laser (Laser Quantum Ltd, England) with an excitation wavelength of $532 \mathrm{~nm}$. Moreover, a highspeed camera (Phantom v7.1, U.S.A.) was connected into the outlet port of the CSU22 (see Figure 2). The microchannel was placed on the stage of the inverted microscope where the flow rate of the working fluid was kept constant at $0.15 \mu \mathrm{l} / \mathrm{min}$ by means of a syringe pump (KD Scientific Inc. U.S.A.). The Reynolds number used in our experiment was 0.014 , whereas the entrance length (Le) was $65 \mu \mathrm{m}$, considering $\mathrm{Le}=1.3 \times($ width/2) $($ Fung 1997). As the measurements were conducted in the middle of the microchannel we have assured that velocity profiles were fully developed at the recording place.

The laser beam was illuminated from below the microscope stage through an air immersion $20 \times$ objective lens with a numerical aperture (NA) equal to 0.75. Satisfactory illumination was achieved by seeding fluorescent particles with $1 \mu \mathrm{m}$ diameter, which absorb green light (absorbance peak $542 \mathrm{~nm}$ ) and emit red light (emission peak $612 \mathrm{~nm}$ ). The light emitted from the fluorescent flowing particles pass through a color filter into the scanning unit CSU22, where by means of a dichromatic mirror is reflected onto a high speed camera to record the PIV images. Although the camera used in this study can record images at a rate of 2000 frames/s the intensity of the images was too dark to be processed by the PIV data analysis, mainly due to low exposure time of the particles. For this reason and because of the complex fluid (PW with $10 \%$ of blood) used in our study we have decided to capture images with a resolution of $640 \times 480$ pixels, 12-bit grayscale, at a rate of $200 \mathrm{frames} / \mathrm{s}$ with an exposure time of $4995 \mathrm{~ms}$. By recording the images for a period of approximately $0.5 \mathrm{~s}$ (100 images for each plane) at a rate of $200 \mathrm{frames} / \mathrm{s}$ the temporal resolution of the measurements was $5 \mathrm{~ms}$. After recording the images they were digitized and transferred to the computer in order to be evaluated using Phantom camera control software (PH607). The PIV images of the flowing particles were processed and the flow velocity was determined by using PivView version 2.3 (PivTec) (Rafael et al. 1998). The images were evaluated by a cross-correlation method, where time between two images was set to $20 \mathrm{~ms}$ and $5 \mathrm{~ms}$ for pure water and blood suspension fluid respectively. By using a multiple-pass interrogation algorithm with 24 by 16 pixel interrogation 
window (vertical and horizontal overlap of $50 \%$ ) which corresponds to a spatial resolution of $28.24 \mu \mathrm{m} \times$ $18.83 \mu \mathrm{m}$, it was possible to obtain the correspondent velocity vector fields.

The confocal micro-PIV system has the unique capability to acquire series of optical sections along $\mathrm{z}$ axis. In order to obtain the optical sectioned images we have used a three dimensional system (RT3D, Yokogawa) which comprises mainly a RT3D signal and low voltage piezoelectric translator (LVPZT) controller. By combining the RT3D system with CSU22 it is possible to generate optical sectioned images with a step size (distance between optical sections, Wright and Wright 2002) as small as $1 \mu \mathrm{m}$ under computer control.

Table 1. Experimental parameters for the present study.

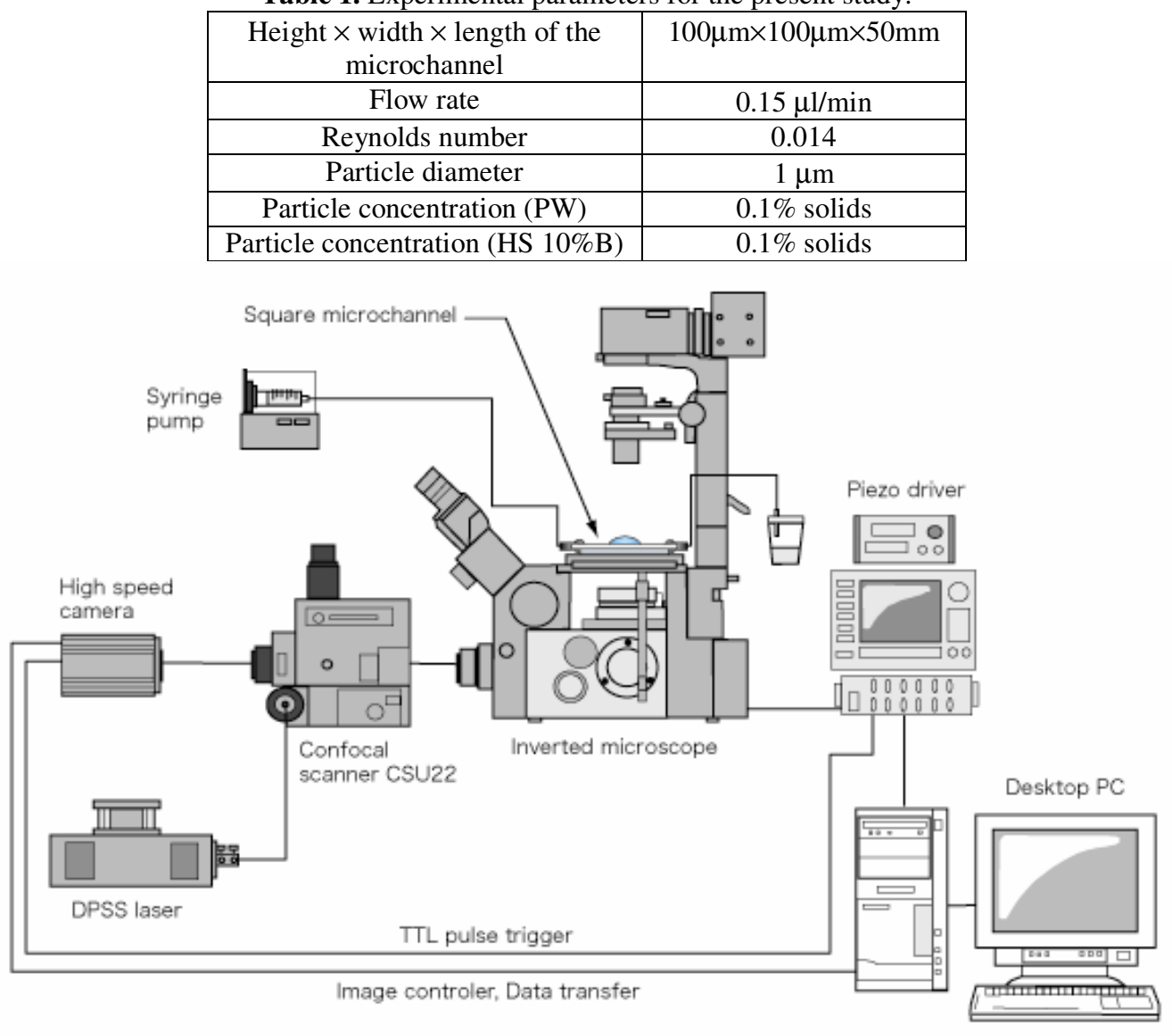

Figure 2. Experimental set-up of the confocal micro-PIV system..

\subsubsection{Spinning disk confocal microscope}

In a conventional microscope the entire depth or volume of the sample is continuously illuminated which leads to the detection of out-of-focus emitted light as well as light from the focal plane of interest. However, with a confocal microscope the sample is scanned with one or more very small spots that illuminate only the plane of focus at one time. In this way, due to the presence of suitable positioned confocal pinholes which act as a spatial filter, out-of-focus emitted light is removed and as a result improves the lateral and axial spatial resolution. This spatial filter is the key to create optical sectioning planes along the depth of the microchannel. In most of the cases, the confocal microscope is combined with a laser light source and scanning system in order to generate the entire field. By repeating a series of scans for multiple focal planes we can obtain at the end a 3D image of the specimen. (Pawley 1989, Wright 2002, Inoue 2002, Park et al. 2004). However, the conventional confocal microscopes uses a point scanning confocal system which requires several seconds to generate a full frame image. This is good enough to scan static specimens but is very slow to study microfluidics phenomena such as blood flow. One way to overcome this speed limitation is by using a spinning disk confocal microscope. This type of confocal microscope employs a spinning disk or Nipkow disk with several thousands of pinholes usually arranged in a helical pattern. Because the disk spins very rapidly (1800 rpm to $5000 \mathrm{rpm})$ it is possible to 
capture images in real time which can be directly viewed by eyes or captured by high speed camera. In brief, the scanning method in CSU units developed by Yokogawa Electric Corporation consists of two disks connected together axially, where the upper disk has 20,000 micro-lenses and lower one, known as Nipkow disk, has also 20,000 matching pinholes with $50 \mu \mathrm{m}$ in diameter. The excitation light that passes firstly through the micro-lenses is focused onto its corresponding pinhole and then progress down to illuminate the focal plane. Emitted fluorescent light from the focal plane is captured by the objective lens and focused back by the same path onto the Nipkow disk containing the pinhole array. Those pinholes remove the out-of-focus light and by means of a dichromatic mirror, located between the two disks, the treated emitted light from the focal plane is reflected onto a high speed camera to build up the image (see Figure 1). As the disks spin very rapidly, thousands of spots of light are scanned simultaneously across the focal plane within a very short time. This produces a very clean image for every video frame and can be directly viewed through eye (Wright 2002, Inoue 2002, Tanaani et al. 2002). The first CSU unit developed by Yokogawa was the CSU10 which operates with a fixed disk speed of $1800 \mathrm{rpm}$ where up to 120 full frame images can be acquired every second (Inoue 2002, Tanaani et al. 2002, Park et al. 2004). However, this scanning rate limits the applications of this type of confocal microscope to study high speed flows. In order to overcome this temporal resolution limitation Yokogawa just recently developed a new scanning unit known as CSU22. The CSU22 enables to choose rotation speed of the Nipkow disk from $1800 \mathrm{rpm}$ to $5000 \mathrm{rpm}$ and consequently it is possible to acquire confocal images up to 2000 frames per second (Inoue 2002, Tanaani et al. 2002). Another very remarkable innovation is the incorporation of a high speed objective lens actuator in order to capture in a very accurate way full frame optical-sectioned images along the depth of the microchannel ( $\mathrm{z}$ axis). Note that, this new function can perform with submicron $\mathrm{z}$ direction resolution and can be controlled via computer. In our experiment we have used this new outstanding scanning system in order to investigate blood fluid mechanics.

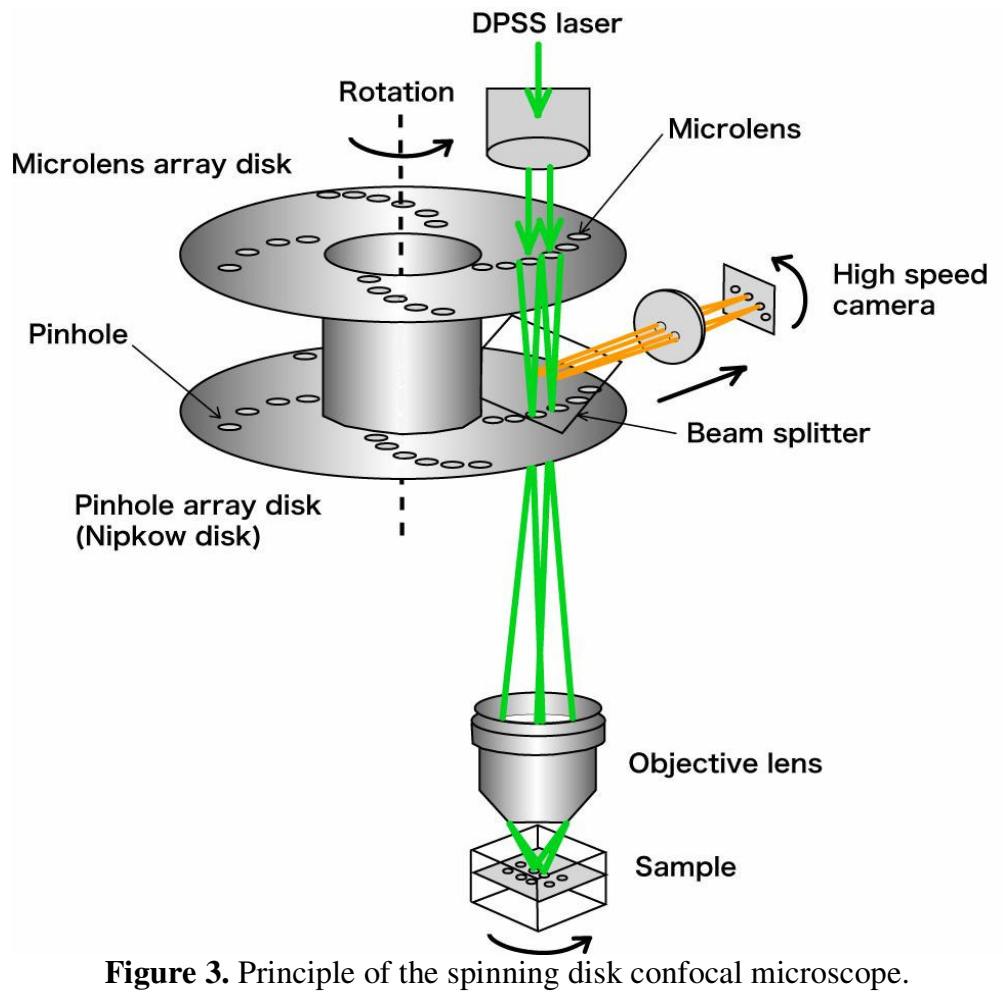

\subsection{Spatial resolution and optical slice thickness of the confocal micro-PIV system}

As already mentioned, spatial filtering done by the pinholes apertures makes optical sectioning possible. This optical sectioning capability enables us to obtain a series of optical sections at different focal planes and as result it is possible to obtain 3D information about the spatial structure of the speciment. It is generally agreed that quality and accuracy of this information depends on the optical section thickness and also on the distance between optical sections (step size). According to Wright 2002, optical section thickness should be smaller than the step size (Wright and Wright 2002, Willhelm et al. 2003). In this way, when using a confocal micro-PIV system it is crucial not only determine the lateral and axial image 
resolution but also the optical slice thickness. Note that for the case of the conventional micro-PIV system the out-of-plane is usually associated to the depth of field (Meinhart et al. 2000, Nguyen and Wereley 2002, Shinohara et al. 2004) whereas in the confocal micro-PIV is related to the optical slice thickness. More detail information about the comparison between conventional and confocal micro-PIV systems can be found elsewhere (Park et al. 2004).

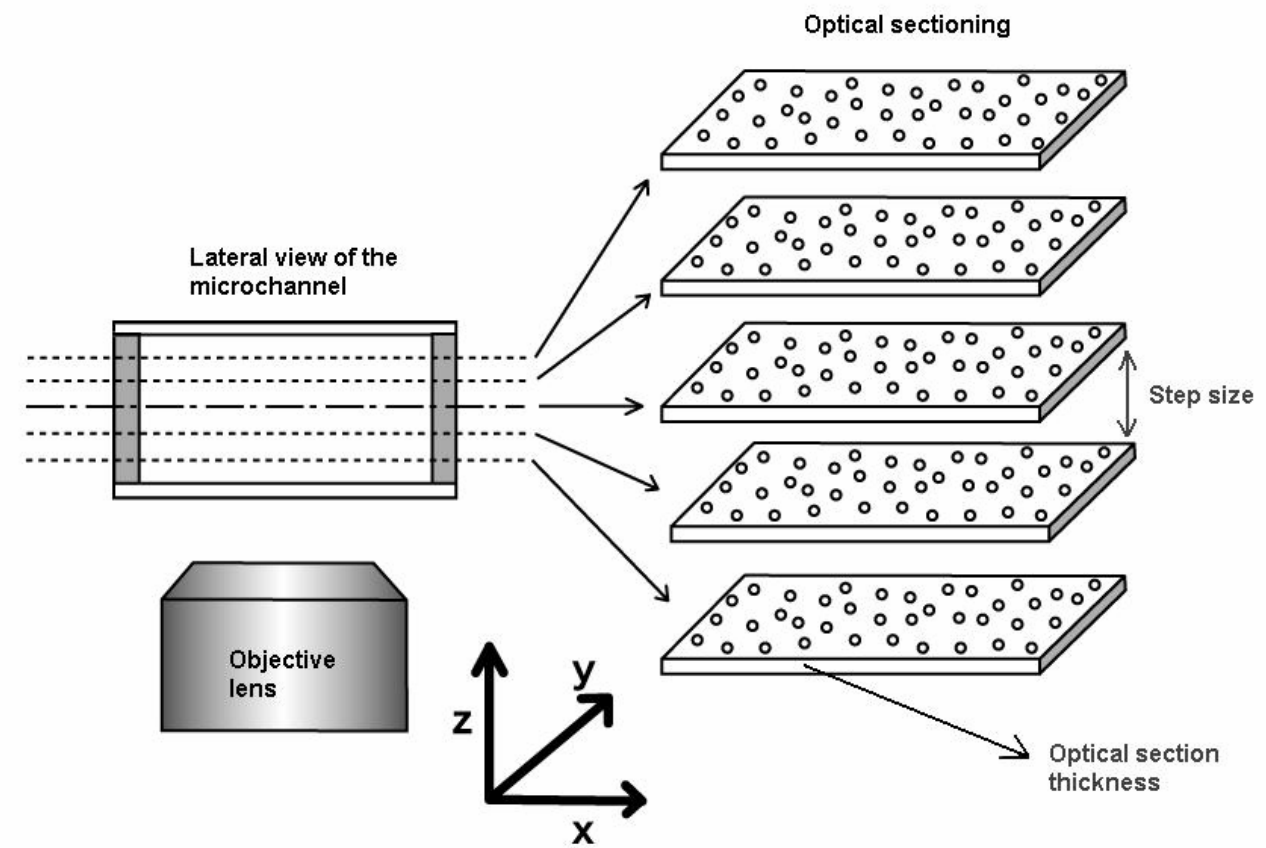

Figure 4. Diagram showing the principle of optical sectioning for PIV measurements.

Confocal microscopy is a well established technique for most biomedical research applications mainly because of its exclusive and unique optical sectioning capability. As a result, analytical expressions for spatial resolution using confocal microscopes are well developed by several researchers such as Inoue 1989, Webb 1996 and Willhelm et al. 2003. One way to obtain lateral resolution, axial resolution and optical slide thickness is by using the concept of resolution probability proposed by Willhelm et al.. Table 1 and 2 show the optical parameters used in the present study and the estimated spatial resolution and optical slice thickness using the equations presented in Appendix A. Note that a full description of the equations presented in the Appendix A can be found at Willhelm et al. 2003.

Table 2. Optical parameters used in the present study.

\begin{tabular}{|c|c|}
\hline$\lambda_{\mathrm{ex}}$ excitation wavelength & $532 \mathrm{~nm}$ \\
\hline$\lambda_{\mathrm{em}}$ emission wavelength & $612 \mathrm{~nm}$ \\
\hline Refraction index (n) & 1 \\
\hline Magnification (M) & $20 \times$ \\
\hline Numerical aperture (NA) & 0.75 \\
\hline Airy unit (AU) & 0.865 \\
\hline Pinhole diameter (PD) & $50 \mu \mathrm{m}$ \\
\hline Modified pinhole diameter (MPD) & $2.5 \mu \mathrm{m}$ \\
\hline
\end{tabular}

Table 3. Spatial resolution and optical slice thickness of the confocal micro-PIV system.

\begin{tabular}{|c|c|}
\hline Lateral resolution $\left(\mathrm{R}_{\mathrm{l}}\right)$ & $0.36 \mu \mathrm{m}$ \\
\hline Axial resolution $\left(\mathrm{R}_{\mathrm{a}}\right)$ & $1.4 \mu \mathrm{m}$ \\
\hline Optical slice thickness $(\mathrm{OST})$ & $4.97 \mu \mathrm{m}$ \\
\hline
\end{tabular}

From the estimated results shown in Table 3 it is possible to verify that our system can achieve very high spatial resolution. For the case of optical slice thickness this parameter can be substantially reduced by increasing the magnification objective (M), NA and n. For instance, by using an oil immersion $60 \times-$ 0.9NA objective lens it is possible to obtain OST less than $1 \mu \mathrm{m}$. However, in our study we have decided 
to use an air immersion 20x-0.75NA objective lens mainly because it has higher working distance and provides a larger field of view and also stronger irradiance onto the light detector. As a result, it was possible to cover the entire width of our microchannel with high spatial resolution and also to obtain adequate quality images along the depth of the square microchannel.

\section{Results}

\subsection{Performance of the confocal micro-PIV}

The confocal micro-PIV system was first evaluated by comparing the experimental results with a well establish analytical solution for steady flow through a long, straight and rigid rectangular microchannel, i. e., Poiseuille flow. In this study, a previously described analytical solution (Bruus, 2004) for a rectangular microchannel is used to compare with the experimental results from the confocal micro-PIV.

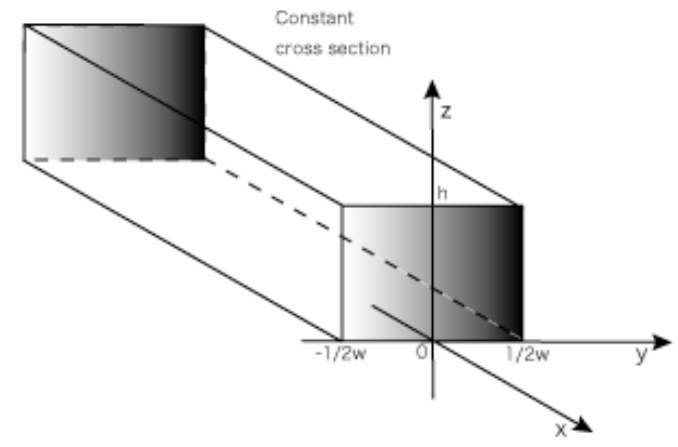

Figure 5. Main parameters of the rectangular microchannel.

By solving the Navier-Stokes equation with a constant pressure gradient along the rectangular microchannel and considering no-slip boundary conditions at the wall the velocity field can be expressed as :

$u_{x}(y, z)=\frac{48 Q}{\pi^{3} h w} \frac{\sum_{n, \text { odd }}^{\infty} \frac{1}{n^{3}}\left[1-\frac{\cosh \left(n \pi \frac{y}{h}\right)}{\left.\cosh \left(n \pi \frac{w}{2 h}\right)\right] \sin \left(n \pi \frac{z}{h}\right)}\right.}{\left[1-\sum \frac{192 h}{n^{5} \pi^{5} w} \tanh \left(n \pi \frac{w}{2 h}\right)\right]}$

This equation is extremely useful mainly when the flow rate is known which is the case of present work. As a result the analytical solutions were determined by considering the flow rate produced by the syringe pump of our experiment. Note that a short survey about how to obtain equation 1 is presented in Appendix B. A full description of this analytical solution can be found at Bruus, 2004.

The determination of the experimental results in the centre plane $(z=h z / 2)$ of the microchannel was performed by using the RT3D system which has sub-micron $\mathrm{z}$ direction resolution. It should be noted that, the origin of the $\mathrm{z}$ axis (lower plate) was set where the fluorescent particles appeared to be almost static.

Figure 6 shows a comparison between theoretical estimations from equation 1 and average fluid velocities of 20 PIV image pairs for a period 0.4s. The PIV measurements were obtained with an exposure time of $4995 \mathrm{~ms}$, magnification factor (pixel/ $\mu \mathrm{m}$ ) 0.85 and time delay of $20 \mathrm{~ms}$ between two images. Note that these results where performed at the middle plane $(50 \mu \mathrm{m}$ height $)$ of the microchannel. According to the results shown in Figure 6, the averaged velocity data obtained from the confocal micro PIV measurements and analytical solution show very close agreement with errors less than $3 \%$. In addition, Figure 6 also confirms that a parabolic Poisseuille flow develops along the square microchannel. 


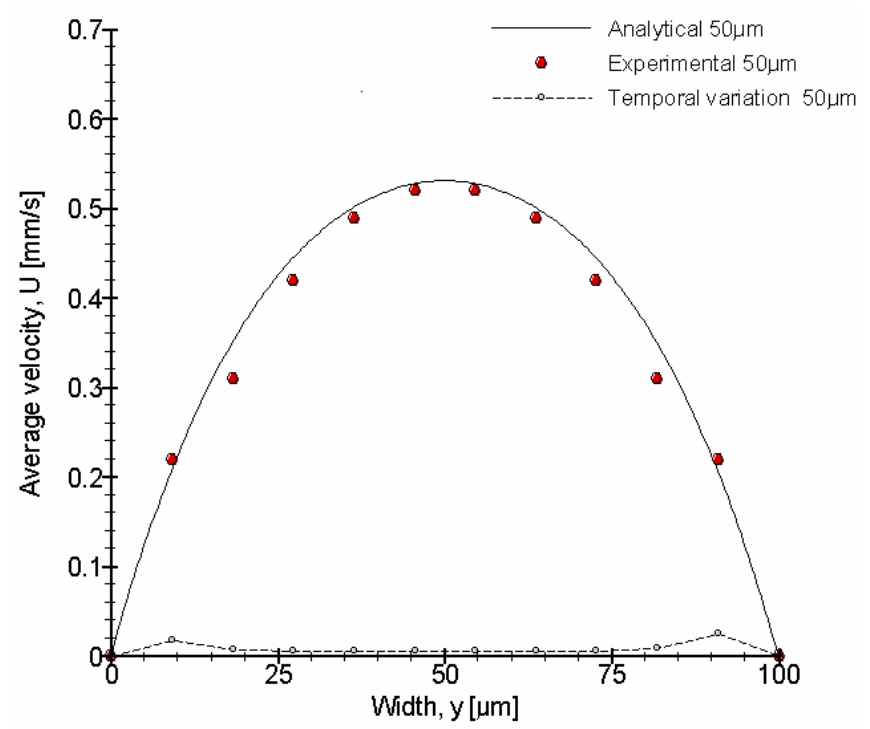

Figure 6.Comparison between experimental data and analytical solution at the centre plane $(50 \mu \mathrm{m}$ height, $\mathrm{Re}=0.014)$.

As already mentioned, the confocal micro-PIV system has the unique capability to acquire series of optical sections along $\mathrm{z}$ axis. In order to analyse the performance of the three-dimensional optical sectioning ability of our system, we have acquired images at several distances from the focal plane. As the theoretical optical slice thickness is approximately $5 \mu \mathrm{m}$, by using a step size of $15 \mu \mathrm{m}$ we ensured that there is any overlap between the optical sectioned images. In Figure 7 the average velocity data are shown for several optical sectioned images along $\mathrm{z}$ axis.

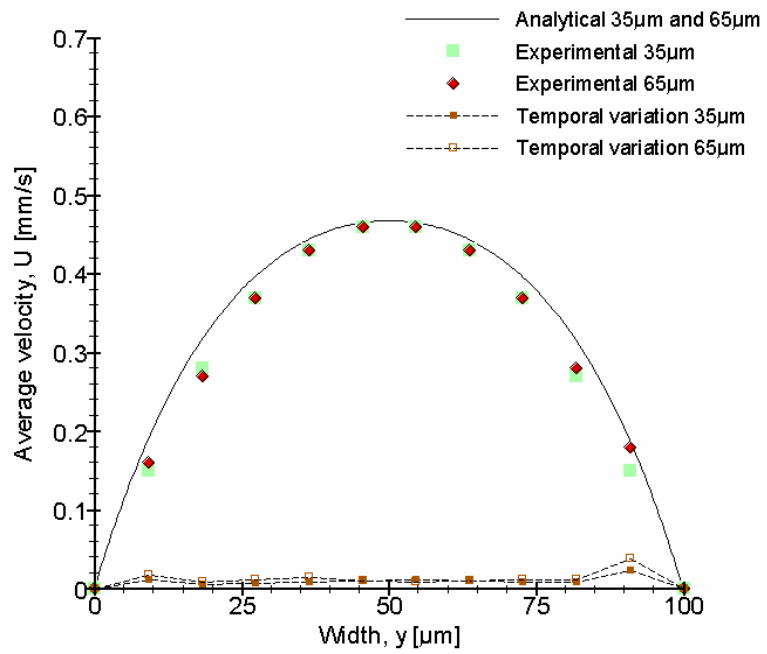

a)

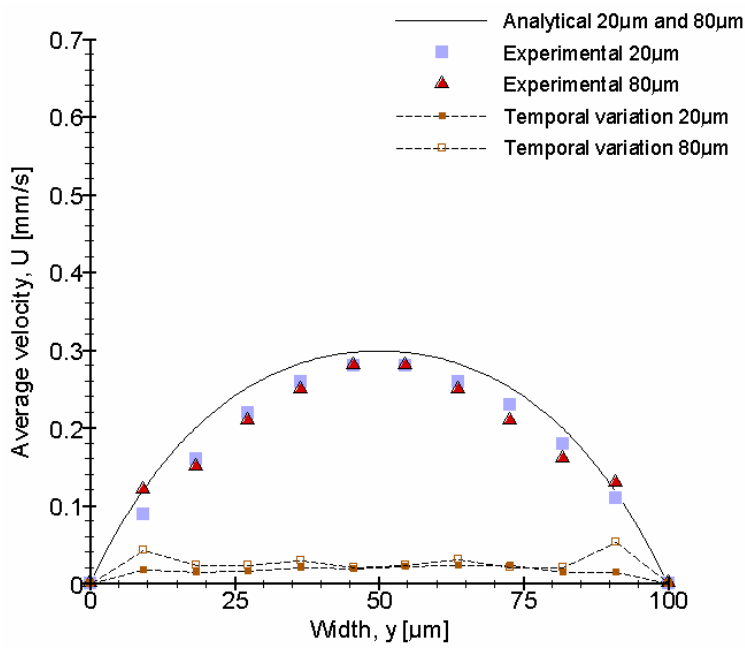

b)

Figure 7. Comparison between experimental data and analytical solution at several optical sectioned images along $\mathrm{z}$ axis; a) comparison at $\mathrm{z}=35 \mu \mathrm{m}$ and $\mathrm{z}=65 \mu \mathrm{m}$ b) comparison at $\mathrm{z}=20 \mu \mathrm{m}$ and $\mathrm{z}=80 \mu \mathrm{m}$.

The results shown in Figure 7a) show very close agreement (errors less than 6\%) with some marginal deviations. However, at locations closer to the wall and far away from the focal plane (xy planes located at $30 \mu \mathrm{m}$ from the centre plane) the deviations were more pronounced with errors from $10 \%$ to $13 \%$.

In order to evaluate the measurement accuracy along the time the temporal variance of the averaged velocities (Uavg) are shown in Figure 6 and 7. Around the middle plane the temporal variations were almost constant with values of about $2 \%$ to $4 \%$ of the time-averaged velocity. These results indicate the ability of our system to obtain high accurate measurements at the centre of the microchannel. On the other hand, near the wall the temporal variations were much higher with values around $0.021 \mathrm{~mm} / \mathrm{s}$ which correspond of about $12 \%$ of the time-averaged velocity. This high variation demonstrates the complexity to obtain very accurate measurements closer to the wall due to some observed background noise presented at the recorded images. 
The analytical solution, for a Newtonian fluid with low Re, within a square microchannel is characterized by one-dimensional flow. By considering the centre of the microchannel as the reference axis, the xy planes located at the same distance from the centre plane must have exactly the same velocities profile. In Figure 7 is possible to observe a fairly good agreement between the average velocity profile at the symmetric $\mathrm{xy}$ planes located at $15 \mu \mathrm{m}(\mathrm{z}=35 \mu \mathrm{m}$ and $\mathrm{z}=65 \mu \mathrm{m})$ and $30 \mu \mathrm{m}(\mathrm{z}=20 \mu \mathrm{m}$ and $\mathrm{z}=80 \mu \mathrm{m})$ from the centre plane.

\subsection{Comparison of velocity profiles of pure water vs blood cells suspension}

As already mentioned, besides the employment of pure water in this study a physiological fluid containing of about $10 \%$ of suspended blood cells was also used. This non-homogenous fluid was selected mainly to evaluate the potentialities of our confocal micro-PIV system to investigate the flow behaviour of complex fluids such as in vitro blood flow.

An example of a recorded image (halogen illumination source) of the physiological fluid used in our experiment is presented in Figure 8 a). In addition, Figure 8 b) shows a confocal image (laser emitted light) used to calculate the velocity field of this non-homogenous fluid. As a result Figure 8 shows the ability of our system to obtain images with just the fluorescent particles within the plasma flow.

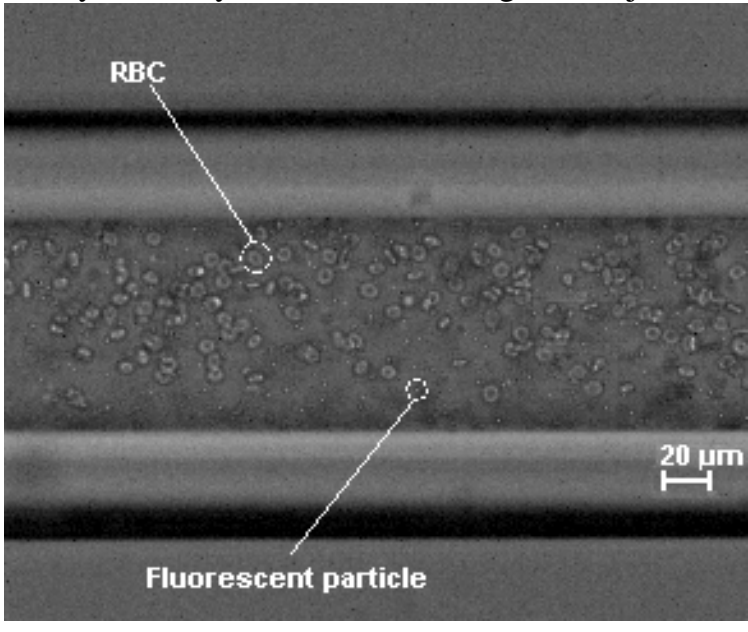

a)

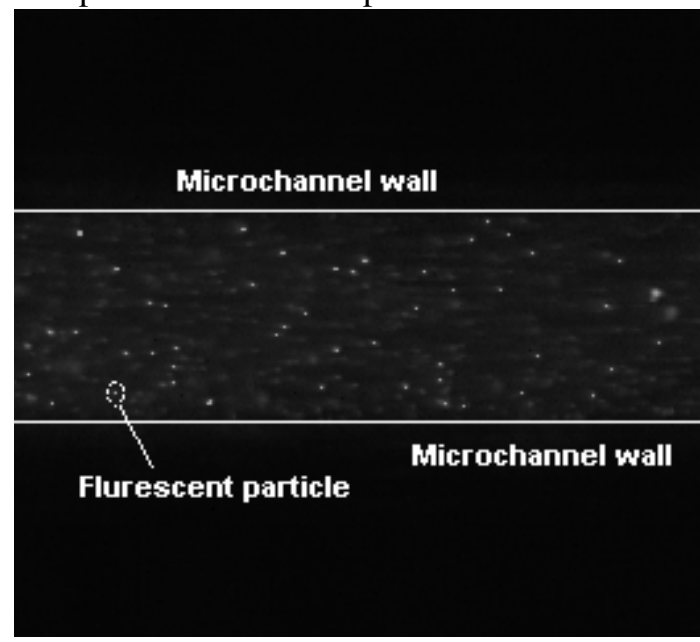

b)

Figure 8 a) Example of an image of the physiological fluid used in this experiment from a halogen illumination source. It is possible to observe both fluorescent particles as very small points $(1 \mu \mathrm{m})$ and RBCs as dark-grey rings.

b) Confocal image from the same physiological fluid where just fluorescent particles are visualized.

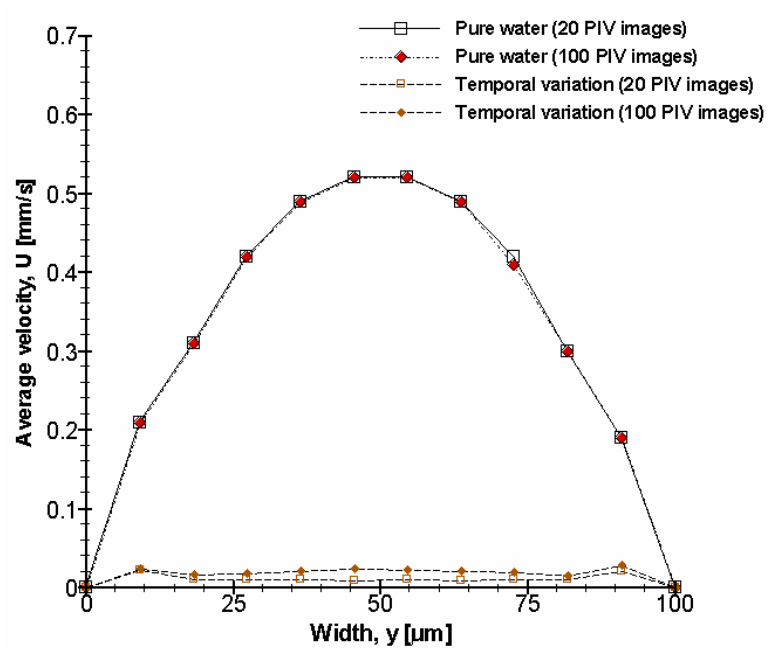

a)

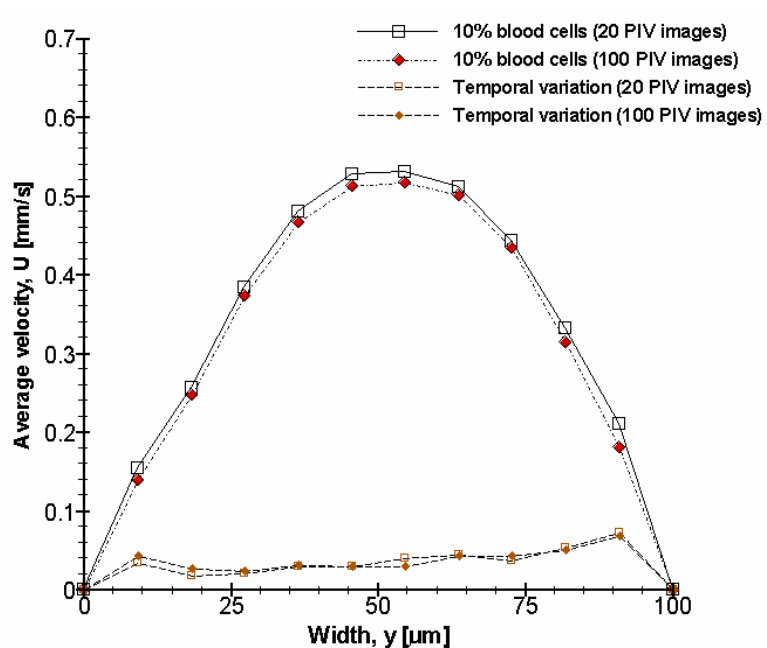

b)

Figure 9. Comparison between 20 and 100 PIV images pairs a) pure water b) $10 \%$ blood cells suspension fluid. 
In order to compare the velocity profiles of pure water and $10 \%$ of a blood cells suspension fluid it is very important to investigate the suitable amount of PIV image pairs required to analyse behaviour of both fluids. Figure 9a) shows the average velocities profiles of pure water for 20 and 100 PIV images pairs with a time delay of $5 \mathrm{~ms}$. For the case of pure water it is very clear that there is almost no difference between the two average velocity profiles which indicates that 20 PIV images pairs are enough to obtain a very reliable description of the flow of an homogenous fluid, such as pure water. However when we made an identical comparison but at this time using a non-homogeneous fluid containing about $10 \%$ of blood cells we have found a slightly difference between the average velocity profiles from 20 and 100 image pairs (see Figure 9b)). As a result, we believe that 100 PIV image pairs with a delay of 5ms will give a more reliable description of the flow behaviour of the non-homogenous fluid used in the present study. Since our main interest is to use the outstanding ability of our system to acquired images at several planes along the microchannel depth, by obtaining more than 100 PIV images in the middle plane it will increase the recording video time and consequently will compromise the measurements of the other planes. Hence, in order to compare the average velocities profiles of both fluids we have decided to use 20 PIV image pairs for pure water and 100 PIV image pairs for the cell suspension fluid with a time spacing between each pair of images of $5 \mathrm{~ms}$.

Figure 9 also compares the temporal variation calculated form the instantaneous velocities of both fluids. An evident increase of the temporal variation of the cell suspension fluid indicates that there are some hydrodynamic disturbance effects into the flow behaviour of the trace particles caused mainly by the presence of blood cells in the flowing plasma.

As a first result, Figure 10 shows the velocity vector fields of pure water and Hanks solution with $10 \%$ of blood, at different xy planes spaced $15 \mu \mathrm{m}$ in depth (z direction).

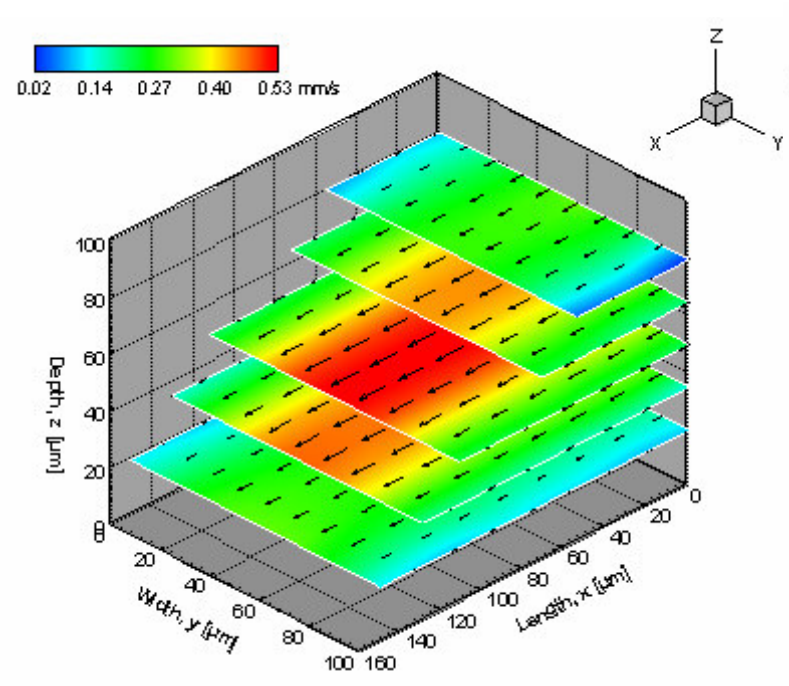

a)

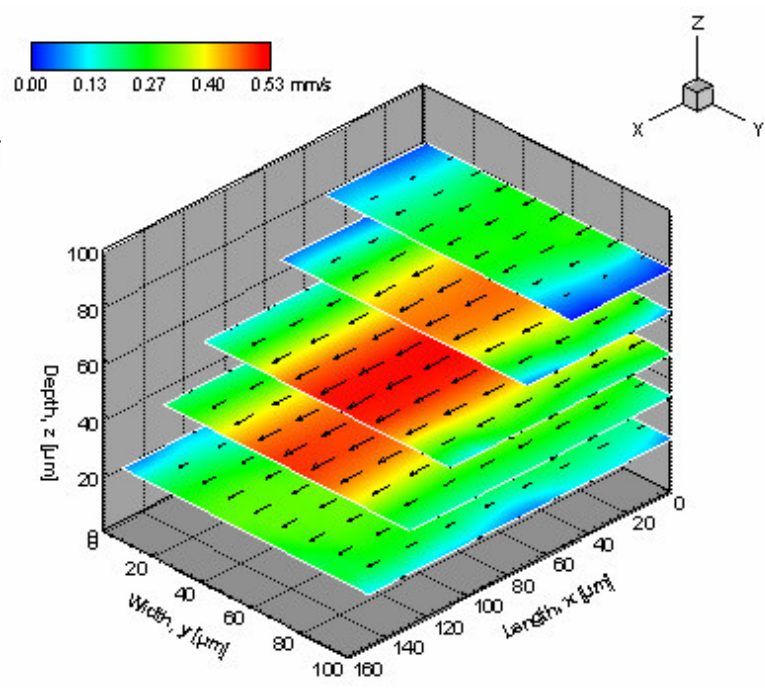

b)

Figure 10. Velocity vector fields at different $\mathrm{z}$ positions of a) pure water and b) $10 \%$ of blood cells suspension

fluid.

From Figure 10a) the velocity profiles of pure water at several horizontal plane (xy axis) show clearly that at this region the flow is stable and fully developed. It is also shown, a close correspondence between the velocity patterns at symmetric xy planes located at $15 \mu \mathrm{m}$ and $30 \mu \mathrm{m}$ from the centre plane. As expected the fluid velocity was highest within the centre of the microchannel whereas at regions very close to the walls the velocities were very small. For the case of the velocity profiles of the physiological fluid (see Figure 10b)) is possible to observe very small deviations between the symmetric planes. This becomes much clearer by analysing the 3D profiles shown in Figure 11. 


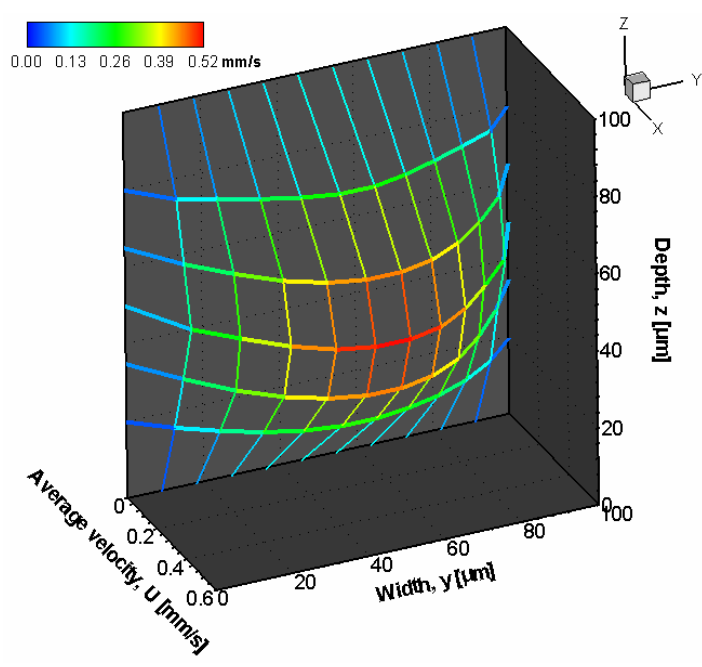

a)

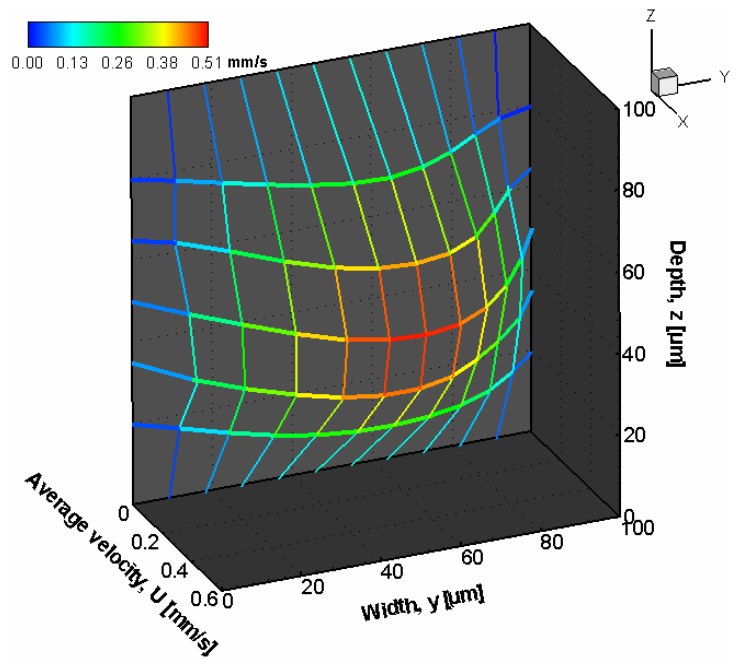

b)

Figure 11. Three dimensional representation of optical sectioning velocity profiles of a) pure water and b) $10 \%$ of blood cells suspension fluid. The 3D profiles were generated from the several optical sectioned images along $\mathrm{z}$ axis measured by the confocal micro-PIV system.

Although the previous ensemble-averaged velocity measurements are known to provide better accurate results in a homogeneous fluid (Santiago et al. 1998, Meinhart et al. 1999, Nguyen and Wereley 2002), when analysing a non-homogeneous fluid some detail information about the flow behaviour could be lost during the ensemble process. This is very clear by comparing the instantaneous velocity fields at the middle plane of both pure water and cell suspension fluid as shown in figure 12 a) and b). Although the instantaneous velocities from pure water have a nearly constant parabolic profile, the instantaneous velocities from the cell suspension fluid exhibit some clear irregularities in the velocity profile. As a result, figure 12b) clearly demonstrates the disturbance effect caused by the blood cells in the instantaneous velocity distribution of the plasma flow. These results are in agreement with the increase of the temporal variation of the cell suspension fluid, as shown in figure $9 b$ ).
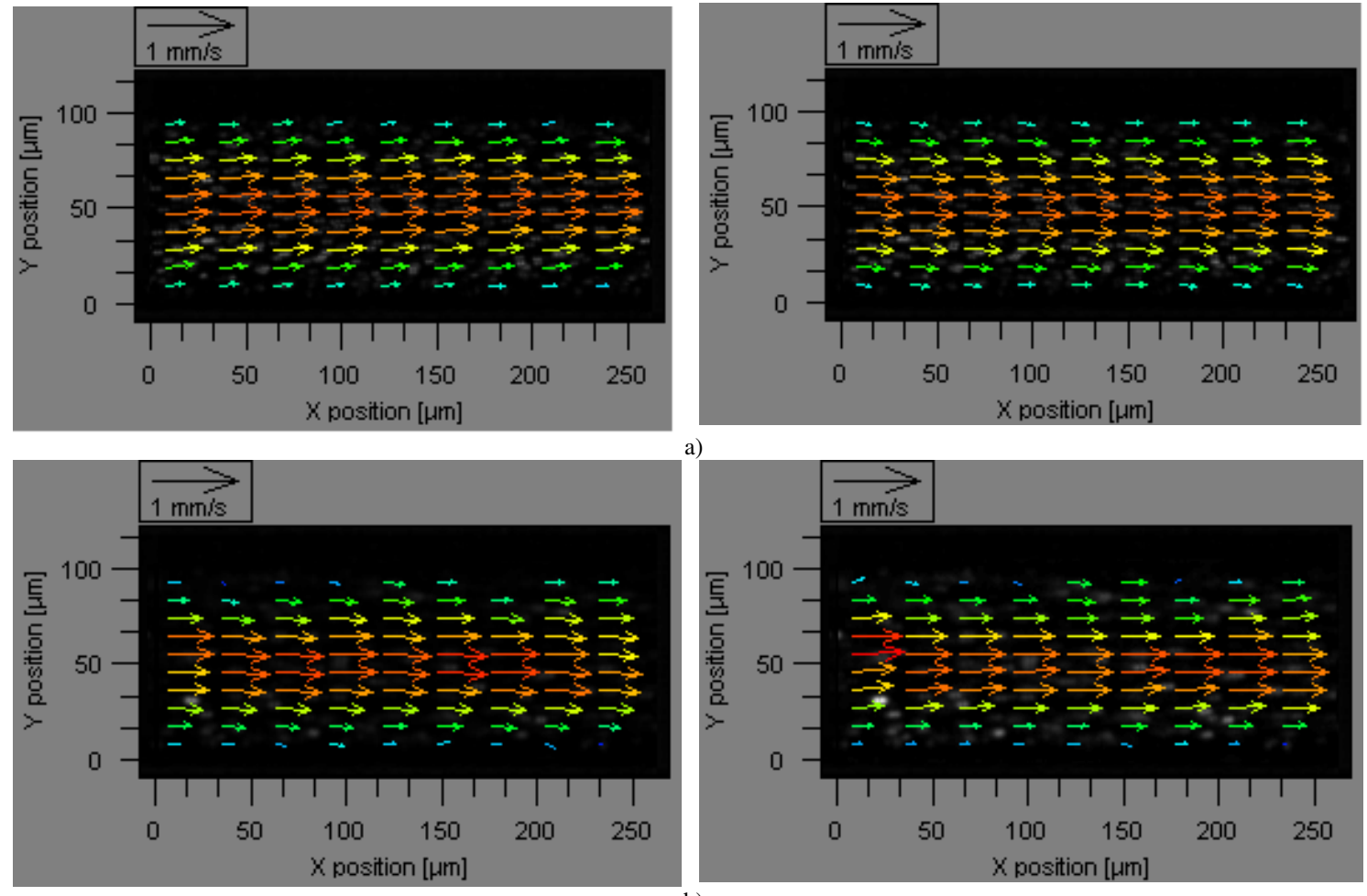

b)

Figure 12. Instantaneous velocities of 2 PIV images pairs with a time delay of $5 \mathrm{~ms}$ a) pure water b) $10 \%$ of blood cells suspension fluid. 


\section{Discusssion}

A very recent study done by Park et al. (Park etal. 2004) has shown, by using a homogenous fluid, the main advantages of the confocal micro-PIV over the conventional micro-PIV. In this present study it is shown the ability of the confocal micro-PIV system to obtain detail measurements of a non-homogenous fluid. Furthermore, due to the new Nipkow disk of Yokogawa, CSU-22, incorporated in our system, we have also shown for the first time the capability of the confocal micro-PIV system to measure velocities up to $0.52 \mathrm{~mm} / \mathrm{s}$ by using a blood cells suspension fluid.

The confocal micro-PIV measurements by using pure water as our working fluid have shown very good agreement when compared with the analytical solution. However, at locations closer to the wall and far away from the focal plane, the errors were slightly bigger than $10 \%$. We believe that this error is mainly due to the increase of the degree of defocusing as one moves out of the ideal focus plane and to "secondorder-effects" such as surface roughness of the wall. Despite this slightly bigger deviations near the wall, the good agreement between micro-PIV measurements and predicted results from the centre planes gives a strong indication that the flow behaviour within $100 \mu \mathrm{m}$ microchannels does follow the macroscale flow theory, which reinforces the recent findings by Meinhart et al (Meinhart et al. 1999) and Devasenathipathy et al. (Devasenathipathy et al. 2003). The results shown here also reinforce the data obtained by Park et al. at a circular micro-tube. Additionally, our results show for the first time that the velocity profiles at symmetric xy planes agree fairly well with predicted Poiseuille profiles. As a result it is possible to conclude that our confocal micro-PIV system has the potentiality to generate threedimensional optical sectioning with high resolution within a range of $100 \mu \mathrm{m}$.

Besides the good agreement with the theoretical predictions, we have also shown that our system at the centre of the microchannel has high measurement accuracy with maximum temporal variations up to $4 \%$ of the time average velocity. However, it is also possible to observe an increase of the temporal variations near the wall of the microchannel. The main possible causes for these higher temporal variations are the increase of the image noise and the high particles diameter used in our experiment. We believe that the measurements close to the wall can be significantly improved by using $200 \mathrm{~nm}$ diameter seeding particles (Meinhart et al. 1999). Note that the employment of $1 \mu \mathrm{m}$ diameter particles was mainly to avoid some possible Brownian motion associated with the low Re used in the present work.

The combination of both optical and experimental parameters used to investigate the behaviour of the flow of a fluid containing suspended blood cells have shown to be a fairly good to obtain quantitative measurements specially at the centre of the microchannel. In fact, it was possible to obtain confocal images with just the fluorescent particles within the plasma whereas the blood cells around the particles were not captured as they had different emission wave lengths. We believe that the clear visualisation of the flowing particles within the suspended cells is mainly due to its ability to obtain extremely thin and in-focus images by means of its unique spatial filtering capability which greatly increase the emitted light and decrease the background noise from the focal plane of interest.

From the comparison of the instantaneous velocities fields of both fluids, it was possible to observe that the velocities profiles from the cells suspension fluid exhibit some small fluctuations caused mainly by the presence of the blood cells within the plasma flow. These fluctuations are in agreement with the increase of the temporal variance of the cells suspension fluid. However, from the ensemble-averaged velocity measurements of the physiological fluid used in the present study, it was possible to observe only very slight deviations when compared with pure water. Hence, these results suggest that $10 \%$ of suspended blood cells have a relatively small effect in the plasma flow and that this physiological fluid behaves as a poiseuille flow. The reason for this behaviour is mainly due to the very small hematocrit used in this experiment. For instance, it was not possible to observe any plasma layer along the flow which is one of main factors contributing to the rheological behaviour of blood flow in microcirculation (Lipowsky, 2005, Mchedlishvili and Maeda 2001). Our results are in accordance with a very recent study carried out by Sugii and his colleagues (Sugii et al., 2005) where they have reported that in vitro blood with low hematocrit $(21 \%)$ behaves as typical Newtonian fluid. However, their work was limited to 2D measurements in the middle of the microchannel. As can be seen from figures 10 and 11, by using our confocal micro-PIV system, it was possible to obtain fairly accurate velocity measurements at several xy 
planes along the $\mathrm{z}$ direction and consequently obtain more detail information about the blood flow behaviour. According to a previous study done by Park and his co-workers (Park et al. 2004), by using a conventional micro-PIV system they have also obtained measurements along the depth of the microchannel, however the accuracy of the measurements was lower when compared with the confocal micro-PIV measurements. The lower accuracy from the conventional micro-PIV is believed to be mainly due to the incapability of the conventional microscopes to obtain true sliced images.

In a very recent study performed by Park et al. they have used a confocal micro-PIV system with a temporal resolution up to 120 frames/s. This low temporal resolution has limited the applicability of their system in microfluidics. In contrast, our system by using the new scanning unit CSU-22 from Yokogawa, allows us to obtain images up to 2000 frames/s, which corresponds to a time resolution of $0.5 \mathrm{~ms}$ between two pairs of images. Although this temporal resolution is possible to be achieved by using pure water, the intensity of the recorded images by using a blood cell suspension fluid was too dark to be processed by the PIV data analysis. By decreasing the temporal resolution to $200 \mathrm{frames} / \mathrm{s}$ and due to the consequent increase of the exposure time of the particles, we have successfully measured velocities up to $0.52 \mathrm{~mm} / \mathrm{s}$. As the velocities in microcirculation are usual from $0.2 \mathrm{~mm} / \mathrm{s}$ up to $10 \mathrm{~mm} / \mathrm{s}$ [Caro et al. 1978, Fung 1997] our system is a very suitable measurement technique to study several phenomena occuring in microcirculation.

It is evident from the present study that the disturbance effect caused by $10 \%$ of blood cells in the plasma flow is relatively small. An attempt to implement our confocal micro-PIV system to investigate the behaviour of in vitro blood with different hematocrits is in its initial stage and now facing some difficulties mainly due to the complex task to control the hematocrit through a microchannel. We expect that by combining the present microchannel with a soft lithography technique to be able to create a bypass flow circuit at the inlet port and as a result to obtain a constant hematocrit through the microchannel.

Finally, although this system has the unique capability to acquire images at several distances from the focal plane, the 3D representation of the axial velocity is still under scope as in this work the velocities along the $\mathrm{z}$ direction are represented from linear relationships between the different optical sectioning velocity profiles. The $3 \mathrm{D}$ velocity profiles could be improved by combining our experimental data with some numerical methods.

\section{Conclusions}

The present study corresponds to the first attempt to obtain detail measurements of a non-homogenous fluid using a confocal micro-PIV system. This emerging technology is a combination of conventional PIV system with a spinning confocal microscope which provides high spatial resolution and 3D flow information. To evaluate the performance of our confocal micro-PIV system, we have investigated the behaviour of two different fluids within a square microchannel at several horizontal planes. The measured velocity profiles of pure water agree well with predicted Poiseuille profiles, which gives indication that microchannels with dimensions of order of $100 \mu \mathrm{m}$ follow the macroscale flow theory. Moreover, the measurements of a physiological fluid containing $10 \%$ of suspended blood cells have demonstrated the ability of this system to obtain confocal images with just the fluorescent particles within the plasma whereas the blood cells were removed due to there different emission wave length. Although it was clearly observed some small fluctuations of the instantaneous velocities fields by comparing pure water with a $10 \%$ of suspended blood cells fluid, when the ensemble-averaged velocities were compared those results indicate that the local disturbance effect caused by the blood cells was not big enough to create significant non-linear effects within the plasma flow. In addition, the results obtain by our confocal micro-PIV system have not only shown the ability of this system to measure velocities up to $0.52 \mathrm{~mm} / \mathrm{s}$ of a blood cell suspension fluid but also the potentiality to generate 3D profiles which allows us to obtain detail information of the flow behaviour within a 100um square microchannel. It should be also stated that this study showed that the confocal micro-PIV system is a very suitable technique to investigate several phenomena of in vitro blood flow in microcirculation. 


\section{Acknowledgments}

This study was financially supported in part by the 21st Century COE Program for Future Medical Engineering based on Bio-nanotechnology, by the International Doctoral Program in Engineering and by a Grant-in-Aid for Scientific Research, No.16200031 and 15086204, from the Ministry of Education, Culture, Sports, Science and Technology of Japan. The authors would also like to thank the technical support provided by Seika Corporation.

\section{References}

Amos W and White J 2003 How the confocal laser scanning microscope entered biological research Biology of the ceel $95 \quad 335-342$

Brown, T 2000 Techniques for mechanical stimulation of cells in vitro: a review Journal of Biomechanics 33 3-14

Bruus, H 2004 Theoritical microfluidics Lecture notes MIC Department of Micro and Nanotechnology Technical University of Denmark Denmark

Caro C, Pedley T, Schroter R and Seed W 1978 The mechanics of the circulation (Oxford University Press)

Chiu J, Chen C, Lee P, Yang C, Chuang H, Chien S and S. Usami S 2003 Analysis of the effect of distributed flow on monocytic adhesion to endothelial cells Journal of Biomechanics 36 1883-1895

Devasenathipathy S, Santiago J, Wereley S, Meinhart C and Takehara K 2003 Particle imaging techniques for microfabricated fluidic systems Experiments in Fluids, 34 504-514

Fung Y 1997 Biomechanics - circulation Second Edition (New York: Springer-Verlag)

Inoue S 1989 Foundations of confocal scanned imaging in light microscopy Handbook of biological confocal microscopy ( Plenum Press) 1-14

Inoue S and Inoue T 2002 Direct-view high-speed confocal scanner: the CSU-10 Cell Biological Applications of Confocal Microscopy (Academic Press ) 87-127

Kinoshita H, Oshima M, Kaneda S, and Fujii T 2005 Confocal micro-PIV measurement of internal flow in a moving droplet, Proc. $9^{\text {th }}$ ICMSCLS (Boston, Massachusetts) 629-631

Klank H, Goranovic G, Kutter J, Gjelstrup H, Michelsen J and Westergaard C 2002 PIV measurements in a microfluidic 3D-sheathing structure with three-dimensional flow behaviour, Journal of Micromechanics and Microengineering, 12 862-869

Koutsiaris A, Mathioulakis D and Tsangaris S 1999 Microscope PIV for velocity-field measurement of particle suspensions flowing inside glass capillaries Measurement Science and Technology 10 1037-1046

Kroll M, Hellums J, Mcintre L, Schafer A and Moake J 1996 Platelets and shear stress Blood 885 1525-1541

Lee J 20001998 Distinguished lecture: biomechanics of the microcirculation, an integrative and therapeutic perspective Annals of Biomedical Eng. 28 1-13

Lima, R. et al., Confocal micro-PIV velocity measurements of physiological fluids in a Rectangular microchannel,

The 3rd IASTED International Conference on Biomechanics - BioMech 2005, IASTED, Benidorm, Spain, CDROM, September, 2005.

Lima R, Wada S, Tsubota K, and Yamaguchi T 2005 Confocal micro-PIV velocity measurements of physiological fluids in a Rectangular microchannel $3^{\text {rd }}$ IASTED ICB - BioMech (Benidorm, Spain) Proc. $\mathbf{4 8 5}$

Lipowsky H 2005 Microvascular rheology and hemodynamics Microcirculation 12 5-15

Mchedlishvili G and Maeda N 2001. Blood flow structure related to red cell flow: a determination of blood fluidity in narrow microvessels Japanese Journal of Physiology 51 19-30

Meinhart C, Wereley S, and Santiago J 1999 PIV measurements of a microchannel flow Experiments in Fluids 27 414-419

Meinhart C, Wereley S and Gray H 2000 Volume illumination for two-dimensional particle image velocimetry Measurement Science and Technology 11 809-814

Nguyen N, and Wereley S 2002 Fundamentals and applications of microfluidics (Norwood, MA: Artech House, Inc.)

Park J, Choi C, and Kihm K, 2004 Optically sliced micro-PIV using confocal laser scanning microscopy (CLSM) Experiments in Fluid 37 105-119

Pawley, J. 1989 Handbook of biological confocal microscopy (New York: Plenum Press)

Raffel M, Willert C and Kompenhans J 1998 Particle image velocimetry: a practical guide (Germany: SpringerVerlag)

Santiago J, Wereley S, Meinhart C, Beebe D and Adrian R 1998 A particle image velocimetry system for microfluidics Experiments in Fluids 25 316-319

Shinohara K, Sugii Y, Arata A, Hibara A, Tokeshi M, Kitamori T and Okamoto K 2004 High-speed micro-PIV measurements of transient flow in microfluidic devices Measurement Science and Technology 15 1965-1970

Sinton D 2004 Microscale flow visualization Microfluid Nanofluid 1 2-21

Sugii Y, Nishio S and Okamoto K 2002 In vivo PIV measurement of red blood cell velocity field in microvessels considering mesentery motion Physiol. Meas. 23 403-416 
Sugii Y, Okuda R, Okamoto K and Madarame H 2005 Velocity measurement of both red blood cells and plasma of in vitro blood flow using high-speed micro PIV technique Measurement Science and Technology 161126 1130

Tanaani T, Otsuki S, Tomosada N, Kosugi Y, Shimizu M and Ishida H 2002 High-speed 1-frame/ms scanning confocal microscope with a microlens and Nipkow disks Applied Optics 4122 4704-4708

Webb R 1996 Confocal optical microscopy Rep. Prog. Phys. 59 427-471

Wilhelm S, Grobler B, Gluch M, and Heinz H 2003 Confocal laser scanning microscopy: principles (Germany: Carl Zeiss)

Wright S and Wright D 2002 Introduction to confocal microscopy Cell Biological Applications of Confocal Microscopy (Academic Press) 1-85

\section{Appendix A. Spatial resolution and optical slice thickness of the confocal micro-PIV system}

The image formed by point light at the focal plane is not an ideal point object, but instead the point is always distributed in a possible area on the image plane. As a result the image properties of the optical system can be described by the point spread function (SPF), which represents the light intensity distribution on the image plane. This distribution has higher probability at the center (higher light intensity) and lower probability at the outside (lower light intensity) (Willhelm et al. 2003).

Resolution can be defined as the ability to distinguish two point objects form each other (Wright and Wright 2002). In confocal microscopy the pinhole plays an important role in calculating its spatial resolution. Considering that modified pinhole diameter (MPD) can be defined as the pinhole diameter (PD in $\mu \mathrm{m}$ ) divided by the magnification (M), and that the airy unit (AU) by the following equation (Willhelm et al. 2003, Park et al. 2004)

$$
A U=\frac{1.22 \lambda_{e x}}{N A}
$$

where $\lambda_{\mathrm{ex}}$ is the wavelength of the illuminating laser light and NA is the numerical aperture of the microscopic objective lens; it is possible to determine the spatial resolution of our system by applying the following criterions:

if MPD > 1.0 AU apply the geometric-optical analysis; if MPD $<0.25 \mathrm{AU}$ apply the wave-optical analysis (Willhelm et al. 2003).

The confocal microscope system used in our experiment follows the geometric-optical analysis, as the $\mathrm{MPD}=2.5 \mu \mathrm{m}$ is bigger than the AU value (AU =0.865). In this way by considering the geometricoptical analysis it is possible to calculate spatial resolution of our system by applying the following equations (Willhelm et al. 2003)

$$
\begin{aligned}
& R_{l}=\frac{0.51 \lambda_{e x}}{N A} \\
& R_{a}=\frac{0.88 \lambda_{e x}}{n-\sqrt{n^{2}-N A^{2}}} \\
& O S T=\sqrt{\left(\frac{0.88 \lambda_{e m}}{n-\sqrt{n^{2}-N A^{2}}}\right)^{2}+\left(\frac{\sqrt{2} n \cdot M P D}{N A}\right)^{2}}
\end{aligned}
$$

where $R_{1}$ is the lateral resolution, $R_{a}$ is the axial resolution, OST is the optical slice thickness, $\lambda_{\text {em }}$ is the emission wavelength and $\mathrm{n}$ the refractive index of immersion liquid. 


\section{Appendix B. Analytical solution for laminar flow in a rectangular microchannel}

By solving the Navier-Stokes equation with a constant pressure gradient along the rectangular microchannel and considering no-slip boundary conditions at the wall, it is possible to obtain following equation for the correspondent boundary conditions (Bruus, 2004)

$\left[\partial_{y}^{2}+\partial_{z}^{2}\right] u_{x}(y, z)=\frac{\Delta p}{\mu L} \quad$ for $\quad-\frac{1}{2} w<y<\frac{1}{2} w, \quad 0<z<h$

$u_{x}(y, z)=0 \quad$ for $\quad y= \pm \frac{1}{2} w, \quad z=0, \quad z=h$

where $\mathrm{u}_{\mathrm{x}}$ is the fluid velocity in the $\mathrm{x}$ direction, $\mathrm{y}$ and $\mathrm{z}$ are the directions normal to the flow, $\mathrm{w}$ is the width of the microchannel, $\mu$ is the fluid viscosity, $\mathrm{L}$ is the length of the microchannel and is the $\frac{\Delta p}{L}$ pressure gradient. Furthermore by using Fourier series along the $\mathrm{z}$ direction it leads to the following velocity field equation as function of location within the cross section of the microchannel and apllied pressure gradient

$u_{x}(y, z)=\frac{4 h^{2} \Delta p}{\pi^{3} \mu L} \sum_{n, \text { odd }}^{\infty} \frac{1}{n^{3}}\left[1-\frac{\cosh \left(n \pi \frac{y}{h}\right)}{\cosh \left(n \pi \frac{w}{2 h}\right)}\right] \sin \left(n \pi \frac{z}{h}\right)$.

In addition by double integration of equation B3 over the cross section, it leads to the correspondent flow rate $(\mathrm{Q})$

$Q=\frac{h^{3} w \Delta p}{12 \mu L}\left[1-\sum_{n, \text { odd }}^{\infty} \frac{192 h}{n^{5} \pi^{5} w} \tanh \left(n \pi \frac{w}{2 h}\right)\right]$

Finally by combining equations B3 and B4 it is possible to obtain a very useful expression to calculate the velocity profile for the Poiseuille flow in a rectangular or square microchannel. In this way the velocity field can then be expressed as:

$u_{x}(y, z)=\frac{48 Q}{\pi^{3} h w} \frac{\sum_{n, \text { odd }}^{\infty} \frac{1}{n^{3}}\left[1-\frac{\cosh \left(n \pi \frac{y}{h}\right)}{\left.\cosh \left(n \pi \frac{w}{2 h}\right)\right] \sin \left(n \pi \frac{z}{h}\right)}\right.}{\left[1-\sum \frac{192 h}{n^{5} \pi^{5} w} \tanh \left(n \pi \frac{w}{2 h}\right)\right]}$ 